\title{
Caracterización clínica de pacientes con Infarto Agudo al Miocardio sin lesiones coronarias significativas. Estudio GEMI 2011-2013
}

\author{
Rodrigo Maldonado ${ }^{1}$, Andrea Madariaga ${ }^{1}$, Constanza López ${ }^{1}$, Carolina Nazzaß ${ }^{3}$, Juan Carlos Prieto ${ }^{2,4}$ \\ 1 Estudiante de Medicina, Facultad de Medicina, Universidad de Chile. \\ 2 Departamento Cardiovascular, Hospital Clínico, Universidad de Chile. \\ 3 Escuela de Salud Pública, Facultad de Medicina, Universidad de Chile. \\ 4 Programa de Farmacología Molecular y Clínica, ICBM, Facultad de Medicina, Universidad de Chile.
}

Antecedentes: El infarto agudo del miocardio (IAM) es consecuencia de la disrupción del endotelio vascular que lleva a oclusión del vaso coronario por un trombo. En la coronariografía de algunos de estos pacientes es posible encontrar arterias coronarias sin lesiones significativas.

Objetivo: Comparar características basales, presentación clínica, tratamiento y mortalidad intrahospitalaria de pacientes con IAM, con y sin lesiones significativas.

Métodos: Se incluyeron 3.194 pacientes registrados en la ficha electrónica GEMI que además del diagnóstico de IAM, hubiesen sido sometidos a estudio coronariográfico. Se consideró "sin lesiones significativas" si ninguna estenosis era $\geq$ al $50 \%$.

Resultados: El grupo sin lesiones significativas correspondió a 116 pacientes $(3,6 \%)$. La edad promedio en este grupo fue menor $(60 \pm 14$ años vs $62 \pm 12$ años, $\mathrm{p}<0,024)$ y con una mayor proporción de mujeres. En este grupo se observó menor prevalencia de dislipidemia $(30,2 \%$ vs $42,8 \%, \mathrm{p}<0.007)$, diabetes mellitus $(14,7 \%$ vs $28,9 \%, \mathrm{p}<0.001)$ y tabaquismo ( $40,5 \%$ vs $61,3 \%, p<0.0001)$. En el ECG de ingreso predominó el IAM sin SDST y menor elevación de CK y CK-MB, junto a una mejor fracción de eyección. Durante la hospitalización se utilizó menos aspirina, clopidogrel, betabloqueadores, heparina ev y HBPM. Existió un mayor uso de antagonistas de Calcio.

Conclusiones: Los pacientes con IAM sin lesiones significativas tienen una menor carga de factores de riesgo coronario, es más frecuente encontrar al ingreso ECG SSDST, menor grado de daño miocárdico y mejor fracción de eyección. Además, se observan diferencias en el tratamiento farmacológico en este grupo de pacientes.

Correspondencia: 


\section{Clinical characteristics of patients with Acute Myocardial Infarction and no significant coronary artery lesions. GEMI Study (2011-1013)}

Background: Acute myocardial infarction (AMI) is a consequence of disruption of coronary artery lesions leading to intracoronary thrombosis. Some patients with AMI show no significant lesions in a coronary angiography.

Aim: To compare clinical characteristics, treatment and intrahospital mortality in patients with AMI with or without coronary artery lesions.

Methods: 3194 patients from a clinical registry of AMI (GEMI) were included. AMI with no significant coronary artery lesions was defined as the abscense of any lesion $\geq 50 \%$.

Results: 116 patients (3.6\%) had no significant coronary lesions (NSL). Compared to the group with significant lesions (SL), they were younger $(60 \pm 14$ years vs $62 \pm 12$ years, $\mathrm{p}<0,024)$ and the proportion of women was higher. NSL patients lower prevalence of hyperli- pidemia ( $30,2 \%$ vs $42,8 \%, \mathrm{p}<0.007)$, diabetes mellitus $(14,7 \%$ vs $28,9 \%, \mathrm{p}<0.001)$ and smoking habit $(40,5 \%$ vs $61,3 \%, \mathrm{p}<0.0001)$. NSL patients had a higher prevalence of non ST elevation AMI and lower levels of $\mathrm{CK}$ and CK-MB, as well as a lower ejection fraction. During hospitalization, NSL patients received aspirin, clopidogrel, betablockers, iv heparin and LMW heparin, less frequently tan SL patients. In contrast, they received calcium antagonists more frequently.

Conclusion: Patients with AMI and no significant coronary lesions have a lower prevalence of coronary artery disease risk factors. A greater frequency of non ST elevation AMI, lower indices of myocardial necrosis and better EF was also observed. The intensity of pharmacologic therapy was lower.

keywords: myocardial infarction, acute; coronary stenosis; hospital mortality 


\section{Introducción:}

Actualmente, está bien establecida la manera en que se diagnostica un Infarto Agudo al Miocardio (IAM), basado en la presentación clínica y los cambios electrocardiográficos y ratificado por la elevación de marcadores de daño miocárdico. ${ }^{1}$

La ruptura de una placa ateroesclerótica en una arteria coronaria inicia una respuesta de agregación plaquetaria, que puede concluir en la oclusión del vaso. ${ }^{2}$ Este proceso trombótico se instala en las arterias coronarias epicárdicas, reduciendo el flujo y disminuyendo la perfusión a nivel de la microcirculación. ${ }^{3}$

Sin embargo, al estudiar mediante coronariografía a estos pacientes, existe la posibilidad de encontrar arterias coronarias sin lesiones significativas, entidad que representa un desafío para su manejo clínico. ${ }^{4}$

La literatura disponible que analiza esta condición, revela una prevalencia entre el $1-12 \%$ del total de infartos, dependiendo de la definición "sin lesiones significativas", que considera desde la ausencia total de estenosis hasta la presentación de lesiones menores al 50\%.5-8

El objetivo de este trabajo es describir y comparar a los pacientes con IAM y arterias coronarias sin lesiones significativas, en relación a: características basales, presentación clínica, tratamiento farmacológico y mortalidad intrahospitalaria. Además, evaluar la asociación entre pacientes con arterias coronarias sin lesiones significativas y factores de riesgo coronario.

\section{Material y métodos:}

Se realizó un estudio transversal de pacientes registrados en la ficha electrónica del Grupo de Estudios Multicéntricos del Infarto (GEMI), en 20 hospitales chilenos durante el período 2011-2013.

Criterios de inclusión: Se incluyeron aquellos pacientes que cumplieran con las siguientes características.

a) Pacientes ingresados con diagnóstico de IAM que cumplieran con al menos 2 de los siguientes criterios: 1) síntomas compatibles con IAM por lo menos de 30 minutos de evolución, 2) cambios ECG en dos derivadas continuas con elevación o depresión del segmento ST o cambios isquémicos en la repolarización ventricular y 3) elevación de marcadores séricos de necrosis miocárdica (troponina $\mathrm{T}$ o I, CK total, CK- MB).

b) Pacientes estudiados con coronariografía durante su hospitalización.

\section{Se dividieron los pacientes en dos grupos:}

- Grupo sin lesiones significativas: pacientes con lesiones coronarias $<50 \%$. No sometidos a trombolisis durante hospitalización y sin antecedentes de cirugía coronaria ni angioplastía previa.

- Grupo con lesiones significativas: Pacientes con al menos estenosis coronaria $\geq 50 \%$.

\section{Variables analizadas:}

a) Características demográficas y factores de riesgo coronario: edad, sexo, hipertensión arterial, dislipidemia, diabetes, tabaquismo y consumo de drogas.

b) Historia cardiovascular: IAM previo, Antecedentes familiares, Angor crónico e Insuficiencia cardíaca.

c) Presentación y evolución del cuadro actual: ECG de ingreso, Killip al ingreso y Fracción de eyección.

d) Marcadores de daño miocárdico: Peak CK, Peak CKMB, Peak Troponina.

e) Tratamiento farmacológico al ingreso, intrahospitalario, y al egreso: Aspirina, Clopidogrel, Betabloqueadores, Estatinas, IECA/ARA II, Antagonistas del calcio, Heparina endovenosa (ev) y Heparina de bajo peso molecular (BPM).

f) Mortalidad intrahospitalaria.

\section{Análisis estadístico:}

Las variables continuas: edad y fracción de eyección, fueron descritas mediante promedio y desviación estándar, y comparadas entre grupos mediante la prueba de $\mathrm{t}$ student. Las variables Peak CK, Peak CK-MB y Peak troponina, fueron descritas a través de la mediana, percentiles $25 \mathrm{y}$ 75 , y comparadas mediante test de medianas. Las variables categóricas fueron comparadas entre grupos mediante test de chi cuadrado. Se aplicó modelo de regresión logística para determinar los factores predictivos de IAM sin lesiones coronarias. Se consideró significativo un $\mathrm{p}<0,05$, y se establecieron intervalos de confianza de $95 \%$. Se utilizó el programa STATA11.

\section{Resultados:}

De los pacientes ingresados a la ficha electrónica GEMI, durante el período 2011-2013, se seleccionaron 3.194 pacientes sometidos a coronariografía durante su hospitalización, de los cuales $116(3,6 \%)$ correspondieron a pacientes sin lesiones coronarias significativas.

Las características demográficas y factores de riesgo se muestran en la Tabla 1. Comparado con el grupo con lesiones significativas, el grupo sin lesiones significativas era levemente más joven (edad promedio $60 \pm 14$ años vs 62 
Tabla 1: Características demográficas y factores de riesgo en los pacientes con IAM comparados según coronarias sin lesiones o con lesiones.

\begin{tabular}{|l|c|c|c|}
\hline \multicolumn{1}{|c|}{ Variable } & Lesiones & Lesiones & \multicolumn{1}{|c|}{$\mathbf{p}$} \\
\hline & $<50 \%$ & $\geq 50 \%$ & \\
\hline & $\mathrm{n}=116$ & $\mathrm{n}=3.078$ & \\
\hline$(\%)$ & $(3,6)$ & $(96.4)$ & \\
\hline Edad (años) & $60 \pm 14$ & $62 \pm 12$ & 0,024 \\
\hline Sexo Femenino \% & 39,7 & 25,2 & $<0.0001$ \\
\hline Hipertensión arterial \% & 65,5 & 68,8 & 0.459 \\
\hline Dislipidemia \% & 30,2 & 42,8 & 0.007 \\
\hline Diabetes Mellitus \% & 14,7 & 28,9 & 0.001 \\
\hline Tabaquismo \% & 40,5 & 61,3 & $<0.0001$ \\
\hline Consumo de drogas \% & 0,86 & 4,2 & 0.077 \\
\hline
\end{tabular}

\pm 12 años $. p<0,024)$ y la proporción de mujeres fue mayor ( $39,7 \%$ vs $25,2 \%, p<0.0001)$. En relación a los factores de riesgo en el grupo sin lesiones significativas, se observó menor prevalencia de dislipidemia (30,2\% vs $42,8 \%$, $\mathrm{p}<0.007)$, diabetes mellitus $(14,7 \%$ vs $28,9 \%, \mathrm{p}<0.001)$ y tabaquismo (40,5\% vs $61,3 \%, \mathrm{p}<0.0001)$.

Con respecto a historia cardiovascular (Tabla 2), en el grupo sin lesiones significativas era menos frecuente el antecedente de IAM $(8,62 \%$ vs $15,9 \%, \mathrm{p}<0,034)$ y más frecuente la historia de angor crónico $(6,9 \%$ vs $3,2 \%$, p $<$ $0.029)$.

\begin{tabular}{|l|c|c|c|}
\hline \multicolumn{3}{|c|}{ Tabla 2: Historia Cardiovascular de los pacientes } \\
con IAM comparada según coronarias sin lesiones \\
o con lesiones significativas.
\end{tabular}

Presentación clínica. En comparación con el grupo con lesiones, el ECG de ingreso predominante en los pacientes sin lesiones significativas fue IAM sin SD del ST $(62,9 \%$ vs $30,1 \%, \mathrm{p}<0,00001)$. Tanto los signos vitales como el Killip al ingreso no presentaron diferencias sustanciales entre ambos grupos. Al analizar los marcadores de daño miocárdico, los valores de CK y CK-MB fueron menores en el grupo sin lesiones significativas. La fracción de eyección fue mayor en los pacientes sin lesiones significativas (Tabla 3).
Tabla 3: Presentación clínica, marcadores de daño miocárdico y fracción de eyección, según coronarias sin lesiones o con lesiones.

\begin{tabular}{|c|c|c|c|}
\hline \multirow[t]{3}{*}{ Variable } & Lesiones & Lesiones & p \\
\hline & $<50 \%$ & $\geq 50 \%$ & \\
\hline & $n=116$ & $n=3078$ & \\
\hline \multicolumn{4}{|l|}{ Presentación clínica. } \\
\hline \multicolumn{4}{|l|}{ ECG de ingreso: } \\
\hline -Con SDST (\%) & 29,3 & 67,8 & 0,00001 \\
\hline -Sin SDST (\%) & 62,9 & 30,1 & 0,00001 \\
\hline -No diagnóstico (\%) & 7,8 & 2,1 & 0,0003 \\
\hline Presión sistólica al ingreso (mmHg) & $143 \pm 28,6$ & $139 \pm 31.1$ & 0,147 \\
\hline Presión diastólica al ingreso $(\mathrm{mmHg})$ & $84 \pm 17,3$ & $81 \pm 18,2$ & 0.038 \\
\hline Frecuencia cardíaca al ingreso (lpm) & $78 \pm 19,4$ & $78 \pm 19,2$ & 0.966 \\
\hline \multicolumn{4}{|l|}{ Killip al ingreso } \\
\hline I (\%) & 85,1 & 79 & 0,147 \\
\hline II (\%) & 7,02 & 14,25 & 0,56 \\
\hline III (\%) & 4,55 & 4,39 & 0,986 \\
\hline IV (\%) & 2,2 & 3,51 & 0,864 \\
\hline \multicolumn{4}{|l|}{ Marcadores de daño miocárdico. } \\
\hline Máximo CK (M; p25-75) & $374 ; 183-712$ & $1219 ; 389-2758$ & $<0.0001$ \\
\hline Máximo CK-mb (M; p25-75) & $51 ; 25-96$ & $123 ; 45-262$ & $<0.0001$ \\
\hline Máximo de Troponina (M; p25-75) & 4,$74 ; 0.6-13,7$ & 5,$51 ; 0,91-25$ & 0,479 \\
\hline Fracción de eyección (\%) & $57 \pm 12$ & $50 \pm 12$ & $<0.0001$ \\
\hline
\end{tabular}

\begin{tabular}{|c|c|c|c|}
\hline \multicolumn{4}{|c|}{$\begin{array}{c}\text { Tabla 4: Tratamientos previos a la hospitalización } \\
\text { en pacientes con IAM. Según coronarias sin } \\
\text { lesiones o con lesiones. }\end{array}$} \\
\hline Tratamiento Previo & Lesiones & Lesiones & $\mathrm{p}$ value \\
\hline & $<50 \%$ & $\geq 50 \%$ & \\
\hline \multicolumn{4}{|l|}{$(\%)$} \\
\hline Aspirina & 37,9 & 56 & $<0.0001$ \\
\hline Clopidogrel & 6 & 33,8 & $<0.0001$ \\
\hline Betabloqueadores & 11,2 & 14,9 & 0.277 \\
\hline Estatinas & 21,6 & 37,9 & $<0.0001$ \\
\hline IECA / ARA I| & 37,1 & 26,1 & 0.008 \\
\hline Antagonistas del Calcio & 2,6 & 5,3 & 0.201 \\
\hline
\end{tabular}

Tratamiento farmacológico. Al analizar el tratamiento previo al ingreso (Tabla 4), en el grupo sin lesiones significativas fue menos frecuente la utilización de aspirina, clopidogrel y estatinas. Además, en este grupo hubo un mayor uso de IECA / ARA II.

Al comparar el tratamiento farmacológico intrahospitalario (Tabla 5), en el grupo sin lesiones significativas existió una menor utilización de aspirina, clopidogrel, betabloqueadores, heparina endovenosa y heparina de BPM, y se 


\begin{tabular}{|c|c|c|c|}
\hline \multicolumn{4}{|c|}{$\begin{array}{l}\text { Tabla 5: Tratamiento intrahospitalario en } \\
\text { pacientes con IAM. Según coronarias sin lesiones } \\
\text { o con lesiones. }\end{array}$} \\
\hline $\begin{array}{l}\text { Tratamiento Previo } \\
\text { (\%) }\end{array}$ & $\begin{array}{c}\text { Lesiones } \\
<50 \%\end{array}$ & $\begin{array}{c}\text { Lesiones } \\
<50 \%\end{array}$ & $p$ \\
\hline Aspirina & 94,8 & 98,6 & 0,001 \\
\hline Clopidogrel & 71,6 & 89,9 & $<0,0001$ \\
\hline Betabloqueadores & 58,6 & 68,2 & 0,030 \\
\hline Estatinas & 94,8 & 93,7 & 0,622 \\
\hline IECA / ARA || & 64,7 & 62,9 & 0.706 \\
\hline
\end{tabular}

\begin{tabular}{|l|c|c|c|}
\hline \multicolumn{4}{|c|}{$\begin{array}{c}\text { Tabla 6: Tratamientos al egreso en pacientes con } \\
\text { IAM. Según coronarias sin lesiones o con lesiones. }\end{array}$} \\
\hline Tratamiento Previo & $\begin{array}{c}\text { Lesiones } \\
\text { IAM }\end{array}$ & $\begin{array}{c}\text { Lesiones } \geq \\
\text { ( }\end{array}$ & $\mathbf{p}$ \\
\hline \multicolumn{1}{|c|}{$(\%)$} & $50 \%$ & \\
\hline Aspirina & 94,8 & 94,4 & 0,826 \\
\hline Clopidogrel & 50 & 80,8 & $<0.0001$ \\
\hline Betabloqueadores & 48,3 & 60,6 & 0,008 \\
\hline Estatinas & 86,2 & 88,9 & 0,375 \\
\hline IECA / ARA II & 58,6 & 50,3 & 0,077 \\
\hline Antagonistas del Calcio & 19,8 & 4,2 & $<0.0001$ \\
\hline
\end{tabular}

utilizaron en mayor proporción los antagonistas de calcio. En relación al tratamiento al egreso (Tabla 6), en el grupo sin lesiones significativas hubo una menor indicación de clopidogrel y betabloqueadores. En cambio, existió una mayor indicación de antagonistas del calcio.

La mortalidad intrahospitalaria, no fue significativamente diferente entre el grupo sin lesiones y el grupo con lesiones ( $2,6 \%$ vs $4,2 \%$, respectivamente, p 0,402$)$.

En el análisis multivariado (Tabla 7), la presencia de IAM sin lesiones significativas se asoció a sexo femenino y a

\begin{tabular}{|c|c|c|c|}
\hline Variable & Odds Ratio (OR) & $\begin{array}{c}95 \% \text { Intervalo de } \\
\text { confianza (IC) }\end{array}$ & $\mathrm{p}$ value \\
\hline Sexo Femenino & 2,24 & $(1,5-3,33)$ & $<0,0001$ \\
\hline Edad & 0,96 & $(0,95-0,98)$ & $<0,0001$ \\
\hline Tabaquismo & 0,34 & $(0,22-0,50)$ & $<0,0001$ \\
\hline Dislipidemia & 0,61 & $(0,40-0,92)$ & 0,02 \\
\hline Diabetes & 0,39 & $(0,23-0,68)$ & 0,001 \\
\hline HTA & 1,12 & $(0,73-1,72)$ & 0,595 \\
\hline
\end{tabular}

menor edad. En cambio, el antecedente de tabaquismo, dislipidemia y diabetes se asociaron a una menor probabilidad de arterias sin lesiones coronarias significativas.

\section{Discusión:}

El infarto agudo al miocardio es una patología cuyo diagnóstico se basa en una presentación clínica característica, cambios electrocardiográficos y aumento de marcadores de daño miocárdico. ${ }^{1}$ Actualmente, la mayor proporción de estos pacientes es sometido a un estudio coronariográfico, ya sea para resolución mediante angioplastía o para diagnóstico de la magnitud del daño coronario. En algunos pacientes la coronariografía revela coronarias sin lesiones significativas, situación que supone un reto en cuanto a su manejo médico. ${ }^{4}$

En este estudio, la prevalencia de IAM sin lesiones significativas en las 3.194 coronariografías efectuadas fue de $3,63 \%$. En la literatura se describe que esta situación es muy variable dependiendo de la publicación y va desde el $1 \%$ al $12 \%$ del total de infartos. ${ }^{5-8}$

En el presente registro, el grupo sin lesiones significativas presentó edad promedio menor que el grupo con lesiones mayores al 50\%, una mayor proporción de mujeres y menor prevalencia de factores de riesgo coronario, como dislipidemia, diabetes mellitus y tabaquismo. Este hallazgo es similar a lo reportado por Larsen y cols., que concluyó que los pacientes con IAM y arterias coronarias normales, fueron más jóvenes y tuvieron menos factores de riesgo para ateroesclerosis tales como hipertensión, hiperlipidemia y diabetes mellitus, en comparación a pacientes con enfermedad coronaria significativa. ${ }^{6}$

Cuando se hace un análisis multivariado para identificar los factores predictores de IAM sin lesiones significativas (Tabla 7), el sexo femenino es la variable que se asocia con mayor fuerza. Otros factores asociados fueron la menor edad, así como la ausencia de tabaquismo, dislipidemia y diabetes mellitus. Al contrastar nuestros resultados con un estudio del año 2008 en España, que incluyó sólo pacientes con IAM sin supra desnivel del ST, se obtuvieron resultados comparables. El sexo femenino fue la variable asociada con mayor potencia a la ausencia de lesiones coronarias significativas. Los otros factores asociados fueron la edad menor a 55 años, la ausencia de diabetes, la falta de tratamiento previo con antiagregantes y la presentación sin infradesnivel del ST en el ECG. ${ }^{7}$

Al comparar los antecedentes de historia cardiovascular, en el grupo sin lesiones significativas se encontró con menor frecuencia el antecedente de IAM previo y mayor probabilidad de historia de angor crónico. No se encuentra 
una clara explicación a este último hallazgo.

En esta población de pacientes, el grupo sin lesiones significativas tuvo un mayor porcentaje de ECG al ingreso sin SDST. Este hallazgo es compatible con síndromes coronarios agudos, donde no existe una obstrucción total del lumen vascular, como en el caso de la angina inestable y el IAM sin supra desnivel del ST.

No hubo diferencias en cuanto a los signos vitales al ingreso ni en el grado de clasificación Killip entre ambos grupos.

En cuanto a la presentación clínica, no existe información detallada en la literatura, aunque se describe en general que no hay diferencias con respecto a los pacientes con lesiones significativas. Por ejemplo, el dolor precordial y los cambios electrocardiográficos serían similares, sin embargo, nuestro registro muestra mayor prevalencia de infarto sin supra desnivel del ST al ECG. ${ }^{5}$

En cuanto a la elevación de marcadores de daño miocárdico, se registraron niveles de CK, CK-MB menores en el grupo sin lesiones significativas. Además, la fracción de eyección medida tanto por ventriculografía como por ecocardiografía, fue de $57 \%$, cifra significativamente mayor a la obtenida en el grupo con lesiones significativas (Tabla 3). En la literatura acerca de IAM con arterias coronarias sin lesiones, se comunica una elevación moderada de biomarcadores de daño miocárdico, un menor grado de isquemia y en consecuencia una menor masa de miocardio comprometido. Además, la función ventricular es usualmente normal $(57 \%) .^{5,8}$

En relación al tratamiento farmacológico previo, en el grupo sin lesiones significativas existió un menor uso de terapia antiagregante plaquetaria, aspirina y clopidogrel. Este hallazgo es similar a lo descrito por Cortell y cols., en una cohorte de pacientes españoles. Respecto al resto de los medicamentos cardiovasculares, en nuestro registro sólo se encontró una menor utilización de estatinas en los pacientes sin lesiones significativas, diferencia que podría explicarse por la menor carga de factores de riesgo coronario en este grupo.

En el grupo sin lesiones significativas, el empleo intrahospitalario de aspirina, clopidogrel, beta-bloqueadores, heparina ev y heparina de BPM fue menor, mientras que los antagonistas de calcio se utilizaron con mayor frecuencia.

Respecto al manejo intrahospitalario, de manera inicial los pacientes ingresados con el diagnóstico de IAM fueron tratados según la terapia establecida por las guías clínicas. Sin embargo, conocida la anatomía coronaria es frecuente suspender el uso de ciertos fármacos, - tal como se cons- tata en este registro -, aunque no existe consenso en un esquema terapéutico particular. ${ }^{9}$

Se podría especular que en algunos casos existe espasmo coronario, entidad secundaria en general a disfunción endotelial que puede ocurrir tanto en vasos normales o, con mayor frecuencia, en vasos con enfermedad. ${ }^{10,11}$ Un $20 \%$ de pacientes con espasmo coronario pueden tener arterias coronarias normales a la arteriografía o enfermedad en grado menor. ${ }^{11}$ Además, una lesión ateroesclerótica menor puede predisponer a vasoespasmo. ${ }^{12}$

Otro grupo de pacientes que presenta angina con arterias coronarias normales a la angiografía corresponde al Síndrome $\mathrm{X}$, entidad cuya definición diagnóstica aún es controversial. ${ }^{13}$ Se acepta en la literatura que el sexo femenino es especialmente propenso a desarrollar Síndrome X durante la edad perimenopáusica (40-50 años). Los mecanismos sugeridos y factores contribuyentes a este síndrome incluyen la disfunción microvascular, alteración de la regulación de la microcirculación y mecanismos de disrregulación autonómica. ${ }^{14}$

El uso de antagonistas del calcio ha beneficiado tanto a los pacientes con angina vasoespástica como a los con espasmo microvascular, donde han demostrado mejoría de la evolución, disminuyendo la injuria miocárdica además de prolongar la sobrevida. ${ }^{15,16}$ Esto explicaría el uso de antagonistas del calcio en más del $20 \%$ de los pacientes del grupo sin lesiones significativas.

El uso de betabloqueadores en este tipo de IAM es controversial. Por una parte se sabe que en el caso de síndrome coronario agudo asociado con el consumo de cocaína, el vasoespasmo coronario empeora en pacientes hipertensos tratados con propranolol, labetalol y esmolol. A su vez, la estimulación alfa-adrenérgica puede agravar el vasoespasmo y la hipertensión. ${ }^{17}$

En general, los bloqueadores beta adrenérgicos están contraindicados por la Sociedad Europea de Cardiología en pacientes con isquemia secundaria a vasoespasmo. Sin embargo, estas recomendaciones no están basadas en evidencia clínica. Tal vez los betabloqueadores que, además, inhiben los receptores alfa, como el labetalol, carvedilol y bucindolol, y aquellos que aumentan el aporte de Óxido Nítrico como nebivolol, puedan ser considerados en la terapia antisquémica de estos pacientes, pero se requiere de ensayos clínicos apropiados para demostrar esta hipótesis. ${ }^{18,19}$

\section{Pronóstico:}

En general los pacientes con IAM y arterias coronarias sin lesiones significativas suelen presentar un excelente pronóstico. Este grupo de pacientes tiene una baja recu- 
rrencia de $\mathrm{IAM}^{20}$ y una tasa de supervivencia a 10 años de un $85 \% .{ }^{21}$

El trabajo de Larsen y cols demostró que aquellos pacientes con arterias coronarias normales tuvieron una tasa de mortalidad de $4 \%$ al año, valor cercano obtenido en pacientes con lesiones coronarias $<50 \%$, mortalidad significativamente menor a la registrada en pacientes con lesiones coronarias significativas. ${ }^{6}$ Por otra parte, en pacientes con IAM sin SDST, Cortell y cols., determinaron que la estenosis coronaria no significativa se asoció a una menor frecuencia de muerte o infarto durante el seguimiento. ${ }^{7}$

En este registro la mortalidad intrahospitalaria fue baja $(4,1 \%)$. Esto podría explicarse por la mejor implementación tecnológica, profesional y la posibilidad de efectuar coronariografía de manera oportuna en estos centros, a diferencia de lo que ocurre en centros que carecen de cardiología intervencional. La mortalidad no difirió significativamente entre ambos grupos (2.6\% en el grupo sin lesiones significativas, vs $4.2 \%$ en los que sí las tenían). Dentro de las limitaciones de este trabajo, podemos señalar que no se evaluó la mortalidad a mediano y largo plazo, a diferencia de los artículos comentados. El número de pacientes sin lesiones significativas podría considerarse bajo, pero es comparable a otros registros publicados. Además, no es posible diferenciar entre lesiones menores al 50\% y coronarias sin ningún grado de lesión, debido a que el instrumento de registro consideró sólo lesiones menores de $50 \%$ de estenosis. Finalmente, ya que la información recolectada es obtenida a través de una encuesta, pueden existir sesgos de información.

\section{Conclusión:}

Podemos aceptar que el IAM en sujetos con arterias coronarias sin lesiones significativas es poco prevalente. Estos pacientes se caracterizan por tener una edad promedio menor en relación a los sujetos con lesiones significativas, en general se asocian a sexo femenino y a la menor prevalencia de factores de riesgo cardiovascular como tabaquismo, dislipidemia y diabetes mellitus. Además, es más frecuente encontrar un ECG de ingreso sin SDST, un menor grado de daño miocárdico evaluado por alza enzimática y una mejor fracción de eyección.

\section{Referencias:}

1. GUARDA E, PRIETO JC, SANHUEZA P, DAUVERGNE C, ASENJO R, CORBALAN R. Guías 2009 de la Sociedad Chilena de Cardiología para el tratamiento del Infarto Agudo del Miocardio con supradesnivel del ST. Rev Chil Cardiol 2009; 28: $223-254$.

2. CHANDRASEKARAN B, KURBAAN AS. Myocardial infarction with angiographically normal coronary arteries. J R Soc Med 2002; 95: 398 - 400.

3. WHITE HD, CHEW DP. Acute Myocardial Infarction. Lancet 2008; 372: 570-84.

4. WANG K, ASINGER R, MARRIOTT H. ST-Segment Elevation in Conditions Other Than Acute Myocardial Infarction. N Engl J Med 2003;349:2128-35.

5. ALPERT JS. Myocardial infarction with angiographycally normal coronary arteries. Arch Intern Med 1994; 154: 265-9
6. LARSEN AI, GALBRAITH PD, GHALI WA, NORRIS CM, GRAHAM MM, KNUDTSON ML. Characteristics and outcomes of patients with acute myocardial infarction and angiographically normal coronary arteries. Am J Cardiol 2005; 95: 261-3.

7. CORTELL A, SANCHISA J, BODÍA V, NÚÑEZ J, MAINARA L, PELLICER M, et al. Infarto de miocardio sin elevación del ST con coronarias normales: predictores y pronóstico. Rev Esp Cardiol. 2009; 62:1260-6.

8. STENSAETH KH, FOSSUM E, HOFFMANN P. Clinical characteristics and role of early cardiac magnetic resonance imaging in patients with suspected ST-elevation myocardial infarction and normal coronary arteries. Int J Cardiovasc Imaging (2011) 27:355-365.

9. AGEWALL S, EURENIUS L, HOFMAN-BANG C, MALMQVIST K, FRICK M, JERNBERG T, et al. Myocardial infarc- 
tion with angiographically normal coronary arteries. Atherosclerosis $2011 ; 219$ : 10-14.

10. MASERI A, SEVERI S, NES MD, et al. "Variant" angina: one aspect of a continuous spectrum of vasospastic myocardial ischemia. Pathogenetic mechanisms, estimated incidence and clinical and coronary arteriographic findings in 138 patients. Am J Cardiol 1978; 42:1019-1035

11. GINSBURG R, SCHROEDER JS, HARRISON DC. Coronary artery spasm--pathophysiology, clinical presentations, diagnostic approaches and rational treatment. West J Med 1982; 136:398-410.

12. CHANDRA S, SINGH V, NEHRA M, AGARWAL D, SINGH N. ST-segment elevation in non-atherosclerotic coronaries: a brief overview. Intern Emerg Med 2011; 6:129-139.

13. VERMELTFOORT I, RAIJMAKERS P, RIPHAGEN I, ODEKERKEN D, KUIJPER A, ZWIJNENBURG A. Definitions and incidence of cardiac syndrome $\mathrm{X}$ : review and analysis of clinical data. Clin Res Cardiol 2010; 99:475-481.

14. JONES E, ETEIBA W, MERZ N. Cardiac Syndrome $X$ and Microvascular Coronary Dysfunction. TCM 2012; 22: 161168.

15. SOLE MJ, LIU P. Viral myocarditis: a paradigm for understanding the pathogenesis and treatment of dilated cardiomyopathy. J Am Coll Cardiol. 1993; 22: 99A-105A.
16. ITO A, FUKUMOTO Y, SHIMOKAWA H. Changing Characteristics of Patients with Vasospastic Angina in the Era of New Calcium Channel Blockers. J Cardiovasc Pharmacol. 2004 ; 44:480-5.

17. LANGE RA, CIGARROA RG, FLORES ED, MCBRIDE W, KIM AS, WELLS PJ, et al. Potentiation of cocaine-induced coronary vasoconstriction by beta-adrenergic blockade. Ann Intern Med. 1990;112:897-903.

18. LÓPEZ-SENDÓN J, SWEDBERG K, MCMURRAY J, TAMARGO J, MAGGIONI AP, DARGIE H, et al. Expert consensus document on b-adrenergic receptor blockers. The Task Force on Beta-Blockers of the European Society of Cardiology. Eur Hear J. 2004;25:1341-62.

19. VIVEK V. BHOSALE, S.C. INAMDAR, KARANDE V.B., BURUTE S.R., MURTHY M.B., GHATAK A. Beneficial Effects of Nebivolol in Comparison with Atenolol on Safety and Tolerability in Essential Hypertension. Journal of Clinical and Diagnostic Research. 2014; 8: HC01-HC041.

20. BETRIU A, PARE JC, SANZ GA, CASALS F, MAGRIÑA J, CASTAÑER A, et al. Myocardial infarction with normal coronary arteries: a prospective clinical-angiographic study. Am J Cardiol 1981; 48: 28-32.

21. RAYMOND R, LYNCH J, UNDERWOOD D, LEATHERMAN J, RAZAVI M. Myocardial infarction and normal coronary arteriography: a 10 year clinical and risk analysis of 74 patients. J Am Coll Cardiol 1988; 11: 471-7. 